\title{
Hydration knowledge of football among Tamil Nadu University players
}

\author{
Aarthi Nandagopal
}

Sport Scientist

Corresponding Author:

Email: aarthipink90@icloud.com

\section{Introduction}

Human body depends on water to survive. Every cell, tissue, and organ in your body needs water to work properly. The goal of drinking during exercise is to prevent excessive ( $>2 \%$ body weight loss from water deficit) dehydration and excessive changes in electrolyte balance to avert compromised performance, because there is considerable variability in sweating rates and sweat electrolyte content between footballers, customized fluid replacement programs are recommended. ${ }^{1}$

Dehydration can cause significant movements of fluid between compartments of water within the body, which can have a large effect on cell functioning. During short-term high-intensity exercise, glycogen from active muscles is broken down into smaller substrates, causing an increase of intracellular osmolality. While there are mechanisms within the body to maintain homeostasis, the increase in osmolality causes high intracellular osmotic pressure, which pushes extracellular water into cells, causing them to swell. This is magnified in longterm high-intensity exercise where there is a higher concentration of metabolic intermediates and a significant decrease in plasma volume. As a result of cell swelling, the body will prioritize anabolic reactions, such as protein and glycogen synthesis, rather than catabolic (Maughan, 2013). Conclusively, water ingestion is vital in optimizing exercise performance. For example, it can improve exercise capacity through maintaining blood volume, which is pertinent in improving thermoregulation and cardiovascular function when a large volume of fluid is being lost as sweat. It is also an effective way to lowering core temperature (Galloway, 1999; Duvillard, 2004). Further, it helps to delay fatigue and prevent injuries that occur with dehydration and high sweat loss, and helps to lower sub-maximal heart rate, heat stress, heat exhaustion, and potentially heat stroke.

Physical Education (PE) is a compulsory subject for about 500,000 school-going youths aged 7-17yrs. PE is usually conducted outdoors between 0800-1030 hrs or 1600-1830hrs and averages 120-300 minutes per week, in compliance with the guidelines issued by the Ministry of Education of India. Participation and training for sport in the outdoors, 2-3 times a week for up to 3 hours per session after curriculum hours is also common. Therefore, a substantial number of young people are exercising in the heat on a daily basis throughout the year and are exposed to heat stress. Inadequate hydration before, during and after exercise puts the health and well- being of India footballers at risk especially when sport training and PE lessons are conducted outdoors under the hot and humid weather conditions of India.

Heat stroke is not directly caused by dehydration, but instead by the rate of the heat production within the body and the capability of the environment to absorb that heat (Duvillard, 2004).For football matches lasting 45 mins or more, the most likely contributors to fatigue are dehydration, sodium loss and carbohydrate depletion. Proper hydration during training or competition will enhance performance, avoid ensuing thermal stress, maintain plasma volume, prevent hypo-natremia, delay fatigue, and prevent injuries associated with dehydration and sweat loss. Internationally recognized bodies including the American College of Sport Medicine (ACSM) ${ }^{1}$ the National Athletic Trainers Association (NATA), ${ }^{2}$ and American Academy of Paediatrics $(\mathrm{AAP})^{3}$ have established hydration guidelines for youths exercising in the heat which is now applied in the group of footballers.

The purpose of this research is to discover the knowledge of hydration levels among university level Tamil Nadu football players.

\section{Hydration and Performance}

When performing exercise in temperature greater then 30 degree $\mathrm{C}$, athletes can become dehydrated through body mass losses between 2 and 7\%, which can have a negative effect on performance. However, the extent of this effect can range anywhere from 7-60\% performance decrement (Maughan, 2010). For instance, when severe enough, dehydration has been known to decrease VO2 max, which results in increased stress on thermoregulation and cardiovascular functioning, increased depletion of glycogen stores, increased metabolite concentration, and weakened psychological drive for exercise (Sawka, 2001).

Little research has been done on the relationship between hydration and performance in sports of highintensity intermittent exercise. Mears et al. (2013) looked at both high-intensity intermittent exercise and continuous exercise and the water consumption that occurred after exercise. There was a greater volume of water consumption found in trials with high-intensity intermittent exercise, which was accompanied by a higher sweat loss so that overall body mass loss was similar in both trials. However, there was a greater fluid replacement in the high-intensity intermittent exercise trials as well as a greater sensation of alleviated thirst 
which was shown by a decrease in sensation of thirst (Mears, 2013).

Owen et al. (2012) assessed the effect different hydration methods would have on soccer skills and performance. The different methods included no-fluid in which participants could not consume fluids, ad-libitum in which participants could consume fluids as they wished and prescribed-fluid in which participants could only consume fluid to balance out a predetermined sweat rate. They found that prescribed intake resulted in the greatest percentage of sweat replaced and that the greatest percent of body mass lost was win the ad libitum and no-fluid trials. Despite these findings, the participants' performances were similar for all trials, which suggests that mild to modest dehydration does not have a debilitating effect on performance (Owen, 2012).

Kurdak et al. (2010) studied hydration during football (soccer) matches as well. For the first match, both teams were provided with water and were not given any hydration instructions. For the second match, one team continued with that condition while the other was given access to both water and a sports drink and instructed that they had unlimited access to those drinks. There were no differences for any of the parameters between the teams for the first match, or between matches for the team that consumed only water. Further, both teams in the second match had similar hydration outcomes through similar consumption and sweat volumes. However, they did find that during the first match, 16 of the 22 players lost more than $2 \%$ body weight and only 55 degree C, $19 \%$ of fluids lost were replaced.

Da Silva et al. (2011) also looked at fluid consumption within soccer players, but studied ad libitum fluid consumption and sweat loss during a match. Players were allowed to consume water ad libitum but were also told to drink $400 \mathrm{~mL}$ of a $6 \%$ carbohydrateelectrolyte drink throughout the game, $200 \mathrm{~mL}$ before the match, and $200 \mathrm{~mL}$ during half time as that was normal match protocol. Despite consuming $800 \mathrm{~mL}$ of fluids and drinking ad libitum, players only replaced about $50 \%$ of sweat losses and had no significant difference in pre- and post-game thirst sensations. Researchers concluded that limited chances to drink during a match must be a contributing factor to poor fluid replacement as well as the fact that many start matches and practices dehydrated (Da Silva, 2011).

Maughan et al. (2010) reviewed studies that looked at dehydration and soccer skill performance. McGregor et al. (1999) determined that when completing a soccer skills test, performance declined when participants were not allowed to consume fluids and was accompanied by a greater percentage of body mass lost. However, when participants were allowed to consume fluids, their performance was sustained. Additionally, Edwards et al. (2007) also administered a soccer fitness test and found similar results (Maughan, 2010).
Pre-exercise hydration has also been highly recommended because despite athletes being properly educated and creating an effective rehydration strategy, they rarely consume enough to prevent dehydration when in the heat. Beginning exercise rehydrated allows for a greater chance at optimal performance. Accounting for individual gastric emptying and intestinal absorption rates still may not account for individual sweat rate losses, so a disparity can still ensue and cause dehydration or gastrointestinal distress (Galloway, 1999). In addition, many researchers have highlighted the importance of athletes understanding their individual sweat rates and hydration needs in order to develop personalized rehydration strategies.

\section{Aim}

The objective of the research is to assess the knowledge of hydration levels of Tamil Nadu university level football players

\section{Materials and Method}

Study Design: Questionnaire study.

Participants: A convenience sampling of 100 healthy football players who were involved with eight Tamil Nadu university level teams (20 female,60 male) between the age of 18-25 years was obtained.

Inclusion Criteria: Football players, 80 Male and 20 Female.

Exclusion Criteria: Football players less than 18 years of age, injured football players.

Administration of the hydration knowledge questionnaire: The hydration knowledge questionnaire was administered to participants. On average, participants took about 20-30 minutes to complete the 29 statements questionnaire. The hydration questionnaire comprised four segments:

i) Participant information (age, sex, schooling levels) and sources of hydration knowledge (nobody;

teacher/coach; parent/guardian; or others);

ii) Exercise pre-hydration statements;

iii) During-exercise hydration statements

iv) Post-exercise hydration statements.

The questionnaire comprised 29 statements; there were 9 statements about 'pre exercise hydration', 12 statements about 'during-exercise' hydration and eight 8 statements about 'post-exercise' hydration. For each hydration statement, participants responded if they strongly agreed with a "yes" or strongly disagreed with a "no". If a statement was correct, a strongly agreed or a "yes" response tallied as correct, and was given a score of 1. An unsure, disagreed or no response is consequently considered as a wrong answer, and given a score of zero. 


\section{Results}

Performance in exercise hydration knowledge questionnaire

Among 100 football players;

1. Pre-exercise hydration knowledge scores are a total of $(5.9 \pm 1.09)$,

2. During exercise hydration knowledge scores are total of $(6.6 \pm 1.7)$

3. Post exercise hydration knowledge scores are a total of $(5.5 \pm 1.1)$.

4. During exercise hydration knowledge will be significantly lower than pre-exercise and Post exercise hydration knowledge in footballers as the final scores interprets.

5. The knowledge of pre and post exercise hydration knowledge is not significantly different in the case of football.

6. On the whole, during exercise hydration knowledge is to be a lack particularly with football players.

7. There is no difference found between the understandings of exercise hydration levels by gender among footballers.

8. But within the winning teams of men and women football players, men's winning team scored a total of 6 out of 9 in pre-exercise hydration knowledge whereas women's winning team scored 4 out of 9 .

9. In the post hydration knowledge there were no gender differences as both the teams scored a total of 6 out of 8 .

\section{Summary}

\begin{tabular}{|l|c|}
\hline Hydration Knowledge & Scores (Mean \pm S.D) \\
\hline Pre-exercise & $5.9 \pm 1.09$ \\
\hline During exercise & $6.6 \pm 1.7$ \\
\hline Post exercise & $5.5 \pm 1.1$ \\
\hline
\end{tabular}

\section{Graph 1: Hydration Knowledge}

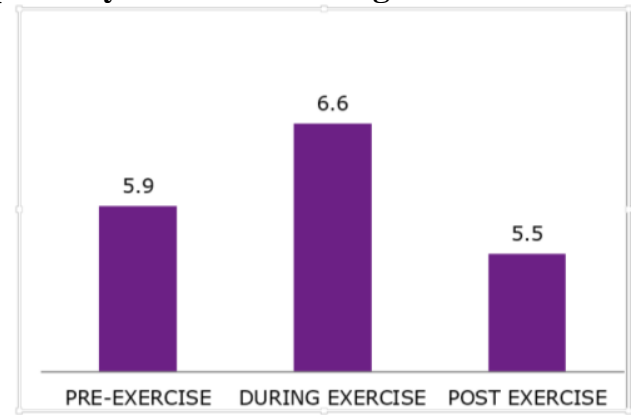

\section{Graph 2: Hydration knowledge of winning team}

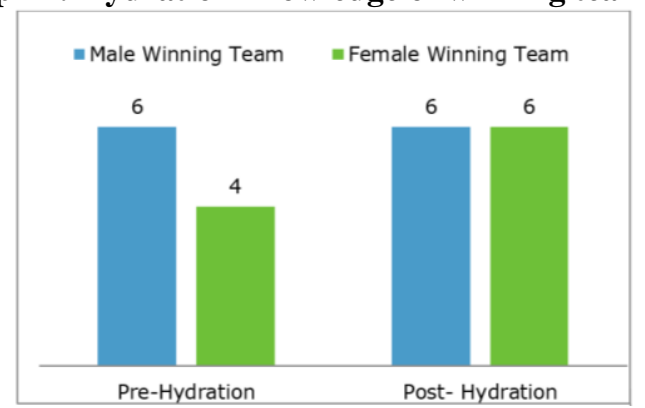

\section{Discussion}

The importance of Indian football players possessing adequate exercise hydration knowledge can be more well educated by having compulsory PE lessons and also sports practice throughout the year. Even though fatalities because of heat stroke among young football players are rare, many non-fatal heat disorders are now reported. Moreover, many experts believe that appropriate knowledge of exercise hydration among youths football players can prevent these heat ailments from occurring in the first instance.

The aim of the present study was to elucidate the exercise hydration knowledge of a large sample of Tamil Nadu University level football players in India. It is noteworthy that the validated exercise hydration questionnaire was developed based upon the position statements of three internationally recognized organizations- ACSM, NATA and AAP - on the subject of exercise hydration and fluid replacement of athletes exercising in the heat. ${ }^{1-2}$ The eventual exercise hydration knowledge questionnaire involved nine statements that relate to pre-exercise hydration, 13 statements that relate to during-exercise hydration and eight statements that relate to post-exercise hydration.

Differences in questionnaire-types on the exercise hydration do not facilitate comparisons across studies, but most of the research has been conducted on nonacclimatized youths from early to late adolescence over the summer months. It appears that the results of the present study represent the first large-scale database of the hydration knowledge and fluid replacement of Indian University level football players.

The key finding was that nearly $80 \%$ of the participants surveyed reported that pre and post exercise hydration were their main sources of exercise hydration knowledge and they were more independent in acquiring their own knowledge of exercise hydration. This is not unexpected, but the results allude to the importance of education about exercise hydration knowledge by coaches and at schools and universities. During the tournament sweat loss was greater than fluid intake. The dehydration was exacerbated by the end of the tournament even though three types of chilled drinks (mineral water, isotonic drink and Milo beverage) were freely available for consumption. 
Adequate exercise hydration knowledge was accepted as a score of $80 \%$ is in keeping with certification standards. While the awareness of during exercise hydration knowledge would be given more importance and counseling sessions by sport nutritionists.

Another key result was that there was not much significant differences in the pre and post exercise hydration knowledge between the winning teams of male and female.

A detailed examination of the data showed that more participants were unsure of the questionnaire statements than those that were answered incorrectly. However, responses to some statements showed that participants answered incorrectly to statements: i) whether athletes should begin exercise well-hydrated; ii) whether athletes should rely on thirst or opportunity to drink for rehydration; iii) whether water is a good hydration fluid for sports events lasting more than an hour; and iv) if a combination of carbohydrate and protein rich food or fluid can help to achieve energy and fluid replacement post-exercise. Thus, in planning for future exercise hydration education programmes, it is crucial that priority and emphasis should be given to address these gaps in knowledge or to reverse such any misconceptions.

\section{Conclusion}

As the results shows the deficit of during hydration knowledge in both Men and Women football players, education about the importance of consumption of required fluid during the sport was very well done with the team after inferring with the given questionnaire. To extend this study with more knowledge related to during sport hydration, sweat trial can be used as a tool to find out the fluid losses which will help infer the requirements of fluid consumption during a sport or a competition and thus a sport nutritionist will guide athletes the necessary amounts of fluid or water to be taken during a completion or sport.

\section{Limitations}

This research was narrowed down to one sport therefore only the exercise hydration knowledge of football were found

\section{References}

1. Armstrong, L.E., Casa, D.J., Millard-Standford, M., Moran, D.S., Pyne, S.W. and Roberts, W.O., American College of Sports Medicine Position Stand: Exertional Heat Illness during Training and Competition, Med Sci Sports Exerc 2007;39(3):556-72.

2. Casa, D.J., Armstrong, L., Hillman, S., Montain, S., Reiff, R., Rich, B., Roberts, W. and Stone, J., National Athletic Trainers' Association Position Statement: Fluid replacement for Athletes, J Athl Training 2000;35:21224.

3. American Academy of Pediatrics [AAP], Council on Sports Medicine and Fitness and Council on School
Health, Policy Statement: Climatic Heat Stress and the Exercising Children and Adolescent, Pediatrics 2011;128(3):e741-e747

4. Bourque, C., Oliet, S., \& Richard, D. (1994).

Osmoreceptors, Osmoreception, and Osmoregulation. Frontiers in Nueroendocrinol 15:231-74.

5. Burdon, C., O'Connor, H., Gifford, J., Shirreffs, S., Chapman, P., \& Johnson, N. (2010).

6. Effect of Drink Temperature on Core Temperature and Endurance Cycling Performance in Warm, Humid Conditions. J Sports Sci 28(11):1147-56. doi:10.1080/02640414.2010.489197 